\title{
氨基修饰的锆基金属有机框架材料对香料的吸附 和缓控释
}

刘宇航 ${ }^{1,2}$, 周珠贤 ${ }^{*}$, 江黎明 ${ }^{2}$, 申有青 ${ }^{1}$

1. 浙江大学化学工程与生物工程学院, 生物质化工教育部重点实验室, 杭州 310027

2. 浙江大学高分子科学与工程学系, 高分子合成与功能构造教育部重点实验室, 杭州 310027

*通讯作者, E-mail: zhouzx@zju.edu.cn

收稿日期: 2018-06-12; 接受日期: 2018-07-26; 网络版发表日期: 2018-11-27

国家重点研发计划纳米科技重点专项(编号: 2016YFA0200300)资助项目

摘要本文以氨基修饰的锆基金属有机框架材料(MOF) UiO-66- $\mathrm{NH}_{2}$ 为香料载体, 研究了其对一系列香料分子 的吸附和释放行为. 我们发现UiO-66- $\mathrm{NH}_{2}$ 对非极性的萜类香料吸附几乎无差别, 而在极性香料的酯类吸附上差异 较大. 我们通过扫描电子显微镜(SEM)、X射线衍射仪(XRD)和红外光谱(FTIR)等对UiO-66- $\mathrm{NH}_{2}$ 吸附香料后的样 品进行表征，结果表明MOF材料吸附香料后不会造成结构坍塌和晶型变化，UiO-66- $\mathrm{NH}_{2}$ 中的氨基与不同的酯类 香料可形成氢键, 对提高吸附量和延长缓释效果起到积极促进作用. 与传统吸附材料活性炭相比, MOF对香料有 更好的吸附效果. 我们使用顶空-气相色谱对酯类香料的释放进行检测, 结合释放动力学模型分析, 发现其释放行 为符合Korsmeyer-Peppas模型, 孔道扩散是其释放的限速性环节.

关键词有机金属框架材料, 香料, 吸附, 控释

\section{1 引言}

众所周知，香料香精产业已广泛应用于食品、日 化和烟草等领域，目前其销售额在世界精细化工大行 业中仅次于医药行业，位居第二位 ${ }^{[1 \sim 3]}$. 随着人们对舒 适生活和高品质芳香享受需求的日益增加，具有抗 菌、镇定和治疗等作用的香料香精产品将在我们的生 活中扮演着越来越重要的作用 ${ }^{[4 \sim 7]}$. 与此同时, 香料本 身不稳定、易挥发等特点，使其不能满足消费者对持 久留香的需求。目前已有的载香方法有微胶囊封 装 ${ }^{[8 \sim 10]}$ 、香料分子与基底材料共价结合 ${ }^{[11]}$ 或者是以聚
合物为包裹外层的潜香体的设计 ${ }^{[12]}$ 以及静电纺丝技 术进行加香 ${ }^{[13]}$ 等. 上述提及的方法大都存在一些缺点 和弊端, 如以微胶囊的载香方法, 因为聚合物微胶囊 在液体环境中胶体稳定差，从而带来制备颗粒尺寸、 控制颗粒形貌和分散性难等问题 ${ }^{[14]}$. 对于聚合物潜香 体或香料分子与基底材料共价结合等方法, 均存在释 放条件苛刻等缺点, 如只能在一定 $\mathrm{pH}$ 、温度、湿度或 光照等条件下进行, 且可供选择的香料分子必须含有 特定的反应官能团, 如氨基、酮基等. 这在一定程度 上也限制了其应用. 对于静电纺丝加香而言，存在的 主要问题是载香量低和材料本身安全隐患等. 因此,

引用格式: Liu Y, Zhou Z, Jiang L, Shen Y. A zirconium-based and amine-functionalized metal-organic framework material for adsorption and controlled release of fragrance. Sci Sin Chim, 2019, 49: 607-612, doi: 10.1360/N032018-00167 
寻找良好的香料载体具有重要意义.

金属有机框架材料(metal-organic frameworks, $\mathrm{MOFs}$ ) 是近十几年内发展起来的由有机配体和金属化 合物通过配位作用自组装形成的新型多孔材料. 目前, 有关MOFs材料在气体储存分离 ${ }^{[15]}$ 、吸附 ${ }^{[16]}$ 、催 化 ${ }^{[17]}$ 、传感器和生物医学 ${ }^{[18]}$ 等领域已有广泛报道, 但 在易挥发分子的控释方面的研究还十分欠缺. Vaughn 等 ${ }^{[19]}$ 利用MOFs材料水稳定差的缺陷，采用湿度诱发 研究了一种疏水香料 $d$-柠檬烯和一种亲水性香料丁酸 乙酯的释放行为. 本文提出通过MOF材料和官能团的 合理修饰来实现香料高封装、可控缓释的目的. 我们 采用水热法 ${ }^{[20]}$ 制备了UiO-66- $\mathrm{NH}_{2}$, 考察了其对非极性 的萜类香料和极性的酯类香料的吸附行为, 并研究了 UiO-66- $\mathrm{NH}_{2}$ 吸附极性酯类香料后的释放行为.

\section{2 实验部分}

\section{1 材料与试剂}

萜类香料 $d$-柠檬烯、 $\alpha$-松油烯和月桂烯以及酯类 香料丙酸乙酯、乙酸丁酯、2-甲基丁酸乙酯等化学药 品都是从安耐吉公司(中国)购买, 所有的化学试剂都 是商业购买且没有进一步纯化.

\section{2 样品表征}

使用岛津XRD-6000粉末衍射仪(日本)对样品进 行晶型表征, 考察其结晶状态, 观察前先对样品通过喷 金制样, 掠角扫描范围为 $3^{\circ} \sim 40^{\circ}$. 使用场发射扫描电子 显微镜 (日立 S 4800 , 日本)和透射电子显微镜(日立 HT7700)对样品微观形貌进行观察. BELSORP-Max (麦奇克拜尔, 日本)全自动吸附仪对UiO-66- $\mathrm{NH}_{2}$ 的孔 结构进行表征和香料吸附量的测定实验. 热重分析仪 (TA, Q50)对样品进行热稳定性分析和香料封装量测 定. 此外, 傅里叶红外光谱(Nicolet iS10, 美国)和气相 色谱(Agilent 7890A, 美国)均有使用.

\section{3 吸附和释放实验}

通过BELSORP-Max有机蒸汽吸附模式实现各类 香料在UiO-66- $\mathrm{NH}_{2}$ 的最大吸附量的测量, 具体实验过 程为向真空的装有固定质量MOF的样品管里缓慢提 供有机蒸汽，使管内压力从 $0 \mathrm{~Pa}$ 增加至香料的饱和蒸 汽压, 最终消耗的有机蒸汽体积换算吸附量. 释放实验
主要考察UiO-66- $\mathrm{NH}_{2}$ 吸附酯类香料后的释放性能，具 体方法为把先吸附好香料的MOF材料放置在顶空瓶 中, 采用 $15 \mathrm{~min}$ 固相微萃取采样方式, 注射入气相色谱 对释放过程进行跟踪表征.

\section{3 结果与讨论}

\subsection{MOF表征}

$\mathrm{X}$ 射线衍射是分析MOF材料的必备手段之一, 图1 为UiO-66- $\mathrm{NH}_{2}$ 与 UiO-66- $\mathrm{NH}_{2}$ 吸附完丙酸乙酯(EP)后 (标记为UiO-66- $\mathrm{NH}_{2} @ \mathrm{EP}$ ) 的XRD图, 可以看到, UiO$66-\mathrm{NH}_{2}$ 在掠角为 $7.8^{\circ}$ 和 $8.6^{\circ}$ 出现特征峰, 分别对应 (111)和(200)晶面, 与之前文献报道的结果一致 ${ }^{[20]}$, 说 明MOF材料本身有很规整的晶体结构. UiO-66- $\mathrm{NH}_{2} @$ $\mathrm{EP}$ 无新峰出现, 出峰位置基本相同, 说明吸附香料后, 并没有破坏其晶体结构, MOF材料内部骨架保持了较 好的完整性. 图2展现的是UiO-66- $\mathrm{NH}_{2}$ 以及UiO-66$\mathrm{NH}_{2} @ \mathrm{EP}$ 的扫描电子显微镜和透射电子显微镜图片, 用来观察样品的微观形貌. 由图2(a)可看到, UiO-66$\mathrm{NH}_{2}$ 是纳米级的多面体结构, 晶体尺寸在200 300 nm, MOF材料具有很好的拓扑结构. 图2(b)展现的是UiO66- $\mathrm{NH}_{2} @ \mathrm{EP}$ 的SEM图, 证明MOF材料结构未发生明显 变化, 与XRD测试结果相符. 图2(c, d) 为透射电子显微 镜观察的UiO-66- $\mathrm{NH}_{2}$ 以及UiO-66- $\mathrm{NH}_{2} @ E$ EP的形貌, 显 示了与上述相同的结果.

图S1 (网络版补充材料)为全自动氮气吸附仪测量 得到的UiO-66- $\mathrm{NH}_{2}$ 的氮气吸附-脱附曲线和孔径分布 图. 结果表明, 吸附曲线是典型的微孔材料I型吸附图, 孔径分布图也证实这一点, UiO-66- $\mathrm{NH}_{2}$ 的孔径集中在 微孔区, 微孔尺寸在 $2 \mathrm{~nm}$ 以下, 主要集中于 $0.5 \sim 0.6 \mathrm{~nm}$. 表 S1(网络版补充材料)为MOF材料的比表面积、孔容 等参数指标. UiO-66- $\mathrm{NH}_{2}$ 的比表面积为 $1000 \mathrm{~m}^{2} \mathrm{~g}^{-1}$ 、 总孔容为 $0.51 \mathrm{~cm}^{3} \mathrm{~g}^{-1}$, 介孔比表面积和孔容均较小, 对 总的比表面积和总孔容贡献极小, 也证实UiO-66- $\mathrm{NH}_{2}$ 的微孔结构特点. 此外, 观察UiO-66- $\mathrm{NH}_{2}$ 的热分析曲 线(图S2)可知, $\mathrm{UiO}-66-\mathrm{NH}_{2}$ 的分解温度为 $265^{\circ} \mathrm{C}$, 其具 有很好的热稳定性和耐受性, 结构不易被破坏.

\section{2 吸附和释放性能的研究}

根据香料极性不同，选取了非极性的天然植物萜 类香料和生活中广泛使用的极性花香、果香味的酯类 


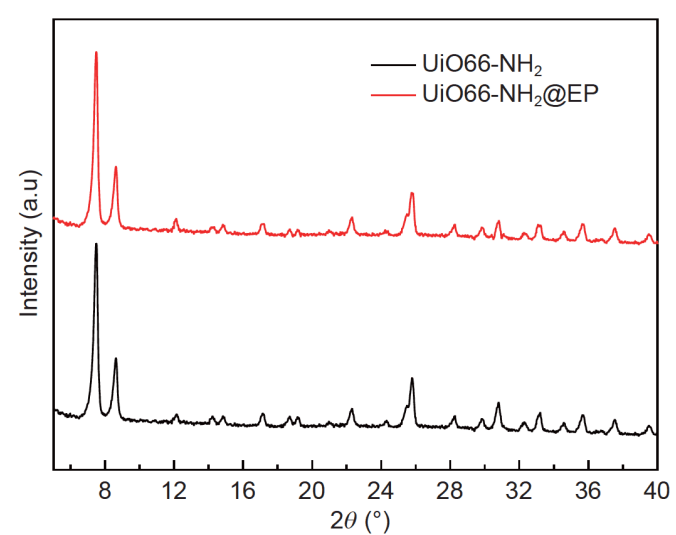

图 1 UiO-66- $\mathrm{NH}_{2}$ 和UiO-66- $\mathrm{NH}_{2} @$ EP的X射线衍射图(网络 版彩图)

Figure 1 X-ray diffraction patterns of $\mathrm{UiO}-66-\mathrm{NH}_{2}$ and UiO-66$\mathrm{NH}_{2} @ \mathrm{EP}$ (color online).

香料作为吸附实验对象. UiO-66- $\mathrm{NH}_{2}$ 对月桂烯吸附量 为 $41.06 \mathrm{~cm}^{3} \mathrm{~g}^{-1}, \alpha$-松油烯吸附量为 $47.27 \mathrm{~cm}^{3} \mathrm{~g}^{-1}$, 柠檬 烯吸附量为 $61.17 \mathrm{~cm}^{3} \mathrm{~g}^{-1}$, 可以看出 3 种非极性香料吸 附差别不大. 而UiO-66- $\mathrm{NH}_{2}$ 对丁酸异戊酯吸附量为 $16.1 \mathrm{~cm}^{3} \mathrm{~g}^{-1}$, 已酸乙酯吸附量为 $38.1 \mathrm{~cm}^{3} \mathrm{~g}^{-1}$, 2-甲基 丁酸乙酯吸附量为 $145 \mathrm{~cm}^{3} \mathrm{~g}^{-1}$, 在这 3 种极性香料上 UiO-66- $\mathrm{NH}_{2}$ 体现出了较为明显的差异. 丁酸异戊酯和 己酸乙酯的吸附量小于非极性香料的原因可以是前两 者极性香料的分子尺寸大于萜类香料，且远大于UiO66- $\mathrm{NH}_{2}$ 的微孔集中分布区域 $(0.5 \sim 0.6 \mathrm{~nm})$, 吸附进入微 孔孔道变得困难, 因此其吸附量较低. 比较分子尺寸近 似但极性不同的柠檬烯和2-甲基丁酸乙酯的吸附可以 看到, UiO-66- $\mathrm{NH}_{2}$ 对极性香料的吸附量是非极性香料
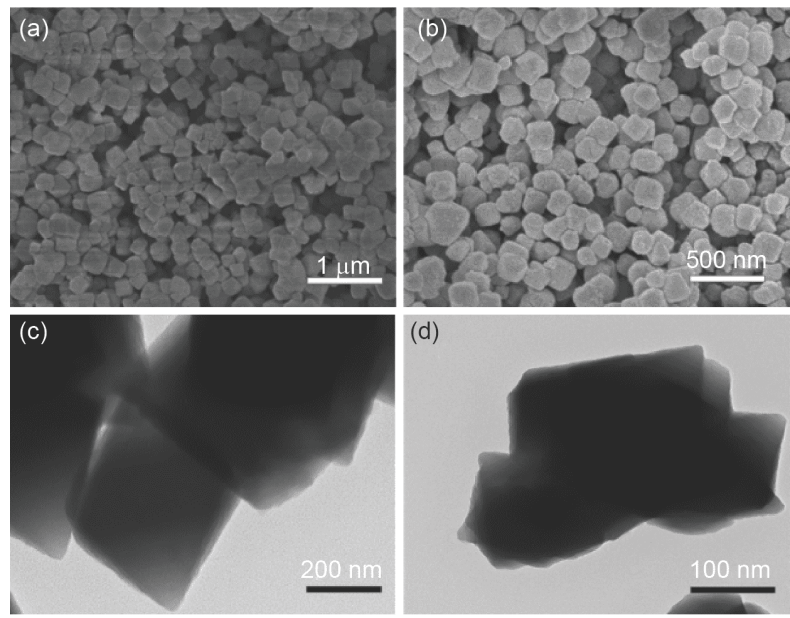

图 2 UiO-66- $\mathrm{NH}_{2}$ 的SEM图(a)和TEM图(c)以及UiO-66$\mathrm{NH}_{2} @$ EP的SEM图(b)和TEM图(d)

Figure 2 SEM image (a) and TEM image (c) of UiO-66- $\mathrm{NH}_{2}$. SEM image (b) and TEM image (d) of UiO-66- $\mathrm{NH}_{2} @ E P$.

吸附量的2 3 倍, 推断UiO-66- $\mathrm{NH}_{2}$ 和极性香料之间似 乎存在一种“极性-极性”的相互关系, 很可能与MOF材 料有机配体中的氨基有关. UiO-66- $\mathrm{NH}_{2}$ 对更多的酯类 香料的吸附数据如表1所示, 其中UiO-66- $\mathrm{NH}_{2}$ 对EP的 吸附量高达 $547 \mathrm{~g} \mathrm{~g}^{-1}$, 体现了极强的吸附能力. 为了对 比其吸附性能, 比较了传统吸附材料活性炭对酯类香 料的吸附情况, 证明了MOF材料是一种优异的加香载 体(表2). 为论证UiO-66- $\mathrm{NH}_{2}$ 和极性香料之间的相互作 用关系, 图3展示的是丙酸乙酯(EP)、UiO-66- $\mathrm{NH}_{2}$ 和吸 附丙酸乙酯后的UiO-66- $\mathrm{NH}_{2} @ \mathrm{EP}$ 的红外光谱谱图, 丙 酸乙酯的甲基和亚甲基在 $2982 \mathrm{~cm}^{-1}$ 附近的伸缩振动

表 1 MOF材料UiO-66- $\mathrm{NH}_{2}$ 对酯类香料的吸附

Table 1 Adsorption of ester fragrance by MOF material UiO-66- $\mathrm{NH}_{2}$

\begin{tabular}{cccccc}
\hline \multirow{2}{*}{ MOF } & \multicolumn{5}{c}{ 香料吸附量 $\left(\mathrm{cm}^{3}(\mathrm{STP}) \mathrm{g}^{-1}\right)\left(\mathrm{g}(\mathrm{FG}) \mathrm{g}^{-1}\right)$} \\
\cline { 2 - 6 } & 丁酸异戊酯 & 己酸乙酯 & 2-甲基丁酸乙酯 & 乙酸丁酯 & 丙酸乙酯 \\
\hline \multirow{2}{*}{ UiO-66- $\mathrm{NH}_{2}$} & 16.1 & 38.1 & 145 & 165.5 & 547 \\
& $(0.114)$ & $(0.246)$ & $(0.847)$ & $(0.85)$ & $(2.49)$ \\
\hline
\end{tabular}

表 2 活性炭与 UiO-66- $\mathrm{NH}_{2}$ 对酯类香料吸附的比较

Table 2 Comparison of adsorption of activated carbon and UiO-66- $\mathrm{NH}_{2}$ on esters

\begin{tabular}{|c|c|c|c|c|c|}
\hline \multirow{2}{*}{ 多孔材料 } & \multirow{2}{*}{ 总比表面积 $\left(\mathrm{m}^{2} \mathrm{~g}^{-1}\right)$} & \multirow{2}{*}{ 总孔容 $\left(\mathrm{cm}^{3} \mathrm{~g}^{-1}\right)$} & \multicolumn{3}{|c|}{ 吸附量 $\left(\mathrm{cm}^{3}\right.$ (STP) $\left.\mathrm{g}^{-1}\right)$} \\
\hline & & & 2-甲基丁酸乙酯 & 乙酸丁酯 & 丙酸乙酯 \\
\hline 活性炭 & 886 & 0.452 & 66.69 & 86.25 & 444.8 \\
\hline UiO-66- $\mathrm{NH}_{2}$ & 1000 & 0.510 & 203 & 180.28 & 587.9 \\
\hline
\end{tabular}


峰在吸附香料后的UiO-66- $\mathrm{NH}_{2}$ 上可见，说明香料已被 成功吸附. UiO-66- $\mathrm{NH}_{2}$ 本身 $3250 \sim 3500 \mathrm{~cm}^{-1}$ 处可归属 于本身氨基官能团，而在UiO-66- $\mathrm{NH}_{2} @ \mathrm{EP}$ 的红外光谱 谱图上，此宽峰在靠近低频数方向有新的小峰出现的 趋势，可解释为氨基中的氢与香料中的羰基氧可能发 生缔合作用产生了氢键，且丙酸乙酯中羰基峰从 $1742 \mathrm{~cm}^{-1}$ 变化到吸附丙酸乙酯的UiO-66- $\mathrm{NH}_{2}$ 的 $1683 \mathrm{~cm}^{-1}$, 同样峰位置有一定位移, 也可以辅证氢键 的产生.

此外，我们也考查了UiO-66- $\mathrm{NH}_{2}$ 作为香料载体的 缓释性能. 采用 UiO-66- $\mathrm{NH}_{2}$ 吸附最好的两种极性酯类 香料: 丙酸乙酯和乙酸丁酯作为释放对象, 先对两种香 料进行吸附实验, 计算得 $5 \mathrm{mg} \mathrm{UiO}-66-\mathrm{NH}_{2}$ 吸附的丙酸 乙酯和乙酸丁酯分别为 9.16 和 $2.16 \mathrm{mg}$. 采用固相微萃 取-气相色谱法表征释放行为, 作为对照实验, 纯EP在 真空瓶中释放对比缓释效果, 纯EP、UiO-66- $\mathrm{NH}_{2} @ \mathrm{EP}$ 和UiO-66- $\mathrm{NH}_{2} @ \mathrm{BA}$ 三者释放曲线如图4所示. 纯EP在 前两天时间里，迅速释放，累计释放量超过 $80 \%$; 在三 天时间完全释放, 释放量接近 $100 \%$. UiO-66- $\mathrm{NH}_{2} @ \mathrm{EP}$ 或@BA展现了较好的缓释效果，半衰期分别约为 3 和 $9 \mathrm{~d}$. Vaughn等 ${ }^{[19]}$ 采用MOFs材料RPM3-Zn对同样为极 性香料的丁酸乙酯(EB)的释放进行了研究，结果表明 RPM3-Zn吸附的EB在 $0.5 \mathrm{~h}$ 时已释放 $80 \%, 1 \mathrm{~h}$ 后基本完 全释放, 缓释效果几乎不存在. 相比而言, $\mathrm{UiO}-66-\mathrm{NH}_{2}$ 对极性香料具有很好的缓释效果. 同样采用药物释放 的动力学方程: 零级释放、一级释放、Higuchi模型和

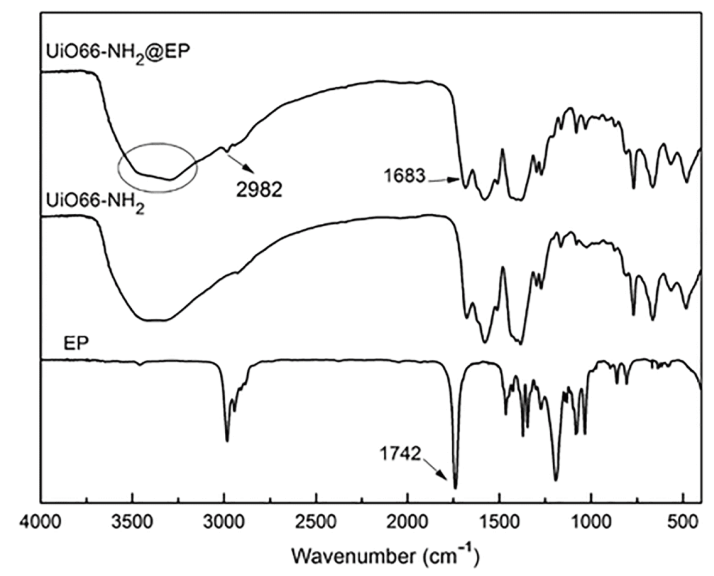

图 3 丙酸乙酯、UiO-66- $\mathrm{NH}_{2}$ 和吸附丙酸乙酯后的UiO-66$\mathrm{NH}_{2}$ 的红外光谱谱图

Figure 3 Infrared spectra of EP, UiO-66- $\mathrm{NH}_{2}$ and UiO-66- $\mathrm{NH}_{2} @ \mathrm{EP}$.
Korsmeyer-Peppas模型分别拟合两种香料释放过程, 结果显示两种香料的释放行为均与Korsmeyer-Peppas 拟合效果最好(图5)，其他拟合结果见表3. KorsmeyerPeppas拟合方程中的 $n$ 值与释放机理相对应 ${ }^{[21]}$ ，当 $n<0.45$ 时, 为扩散控制释放, 当 $n>0.89$ 时, 为溶蚀释放; 当 $0.45<n<0.89$ 时，为扩散和溶蚀协同作用. 本文中两 个 $n$ 值分别为 0.21 和 0.28 , 所以可解释为香料的释放过 程为扩散释放, 孔道扩散为其限速性环节.

\section{4 结论}

本研究应用MOF材料UiO-66- $\mathrm{NH}_{2}$ 作为一种加香 载体, 考察了其吸附香料和香料释放的行为特点. 相比 于传统吸附材料, UiO-66- $\mathrm{NH}_{2}$ 在香料吸附量上有明显 优势, 可以针对特定的如极性香料, 合成含有极性官能 团的MOF能够提高吸附能力. 释放实验表明, UiO-66$\mathrm{NH}_{2}$ 载香的释放过程为扩散释放, 孔道扩散为其限速 性环节。

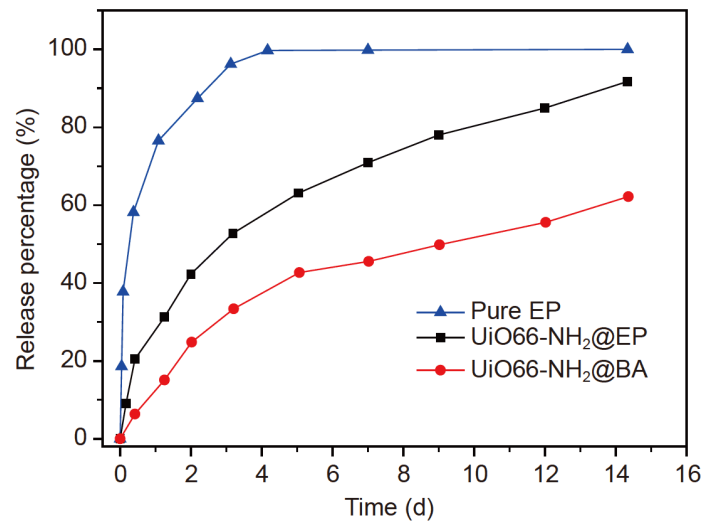

图 4 纯EP、 UiO-66- $\mathrm{NH}_{2}$ 吸附丙酸乙酯与乙酸丁酯后的释 放曲线(网络版彩图)

Figure 4 Release curves of pure EP, UiO-66- $\mathrm{NH}_{2} @ \mathrm{EP}$ and UiO-66$\mathrm{NH}_{2} @ \mathrm{BA}$ (color online).

表 3 各释放动力学方程对UiO-66- $\mathrm{NH}_{2}$ 吸附丙酸乙酯和乙 酸丁酯的释放拟合

Table 3 Release kinetics equations for UiO-66- $\mathrm{NH}_{2} @ \mathrm{EP}$ and UiO66- $\mathrm{NH}_{2} @ \mathrm{BA}$

\begin{tabular}{ccc}
\hline & UiO-66- $\mathrm{NH}_{2} @ \mathrm{EP}$ & UiO-66- $\mathrm{NH}_{2} @ \mathrm{BA}$ \\
\cline { 2 - 3 } & 拟合系数 $R^{2}$ & 拟合系数 $R^{2}$ \\
\hline 零级释放 & 0.846 & 0.859 \\
一级释放 & 0.971 & 0.962 \\
Higuchi 释放 & 0.981 & 0.970 \\
\hline
\end{tabular}



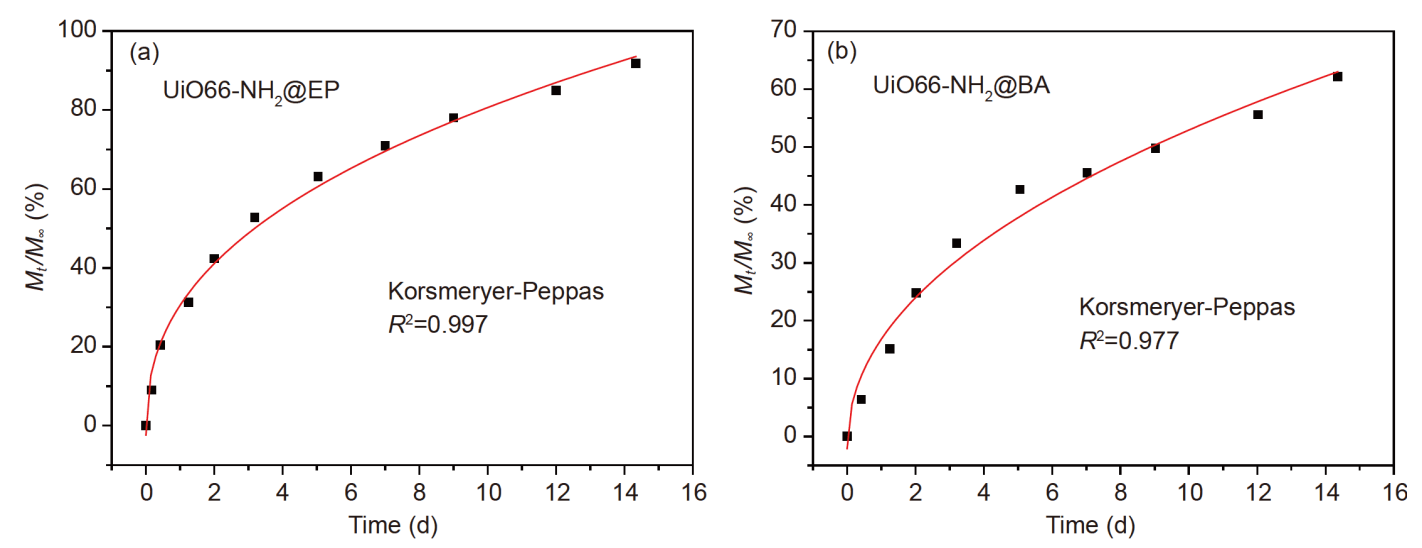

图 5 Korsmeyer-Peppas方程拟合UiO-66- $\mathrm{NH}_{2}$ 吸附丙酸乙酯和乙酸丁酯的释放(网络版彩图)

Figure 5 Korsmeyer-Peppas equation for the release of UiO-66- $\mathrm{NH}_{2} @ \mathrm{EP}$ and UiO-66- $\mathrm{NH}_{2} @ \mathrm{BA}$ (color online).

致谢感谢新加坡国立大学赵丹教授提供 $\mathrm{MOF}$ 材料 $\mathrm{UiO}-66-\mathrm{NH}_{2}$.

\section{补充材料}

本文的补充材料见网络版http://chemen.scichina.com. 补充材料为作者提供的原始数据, 作者对其学术质量和内容负责.

\section{参考文献}

1 Cao L, Qin L, Kang S, Li X. Guangzhou Chem Ind, 2017, 45: 17-20 (in Chinese) [曹琳, 秦利霞, 康诗钊, 李向清. 广州化工, 2017, 45: 17-20]

2 Xiao Z, Hu J, Niu Y, Zheng C, Lu X, Zhu G. J Tech, 2017, 1: 6-9 (in Chinese) [肖作兵, 胡静, 牛云蔚, 郑承臻, 陆欣宇, 朱广用. 应用技术学报, 2017, 1: 6-9]

3 Lu X, Wang H, Zhu W. Fine Chem, 2013, 11: 1234-1236, 1246 (in Chinese) [陆欣宇, 王慧辰, 朱为宏. 精细化工, 2013, 11: 1234-1236, 1246]

4 Liu B, Chai L, Lin X, Bai M. Flavour Frag Cosmetic, 2016, 5: 25-30 (in Chinsese) [刘布鸣, 柴玲, 林霄, 白禁嘉. 香料香精化妆品, 2016, 5: 2530]

5 Qin S, Hu H. J Southeast Univ (Med), 2018, 1: 165-168 (in Chinese) [秦思, 胡怀东. 东南大学学报(医学版), 2018, 1: 165-168]

6 Zhou H, Chen Y, Luo Q. Mod Horticult, 2006, 5: 6 (in Chinese) [周辉明, 陈燕, 罗庆国. 现代园艺, 2006, 5: 6]

7 Lu Z, Zheng Y, Zhang T, Shen J, Xiao Z, Hu J, Niu Y, Yu D, Zhang X. J Biomed Nanotechnol, 2018, 14: 1675-1687

8 Guo J, Duan X. Flavour Frag Cosmetic, 2010, 2: 26-27 (in Chinese) [郭俊华, 段秀珍. 香料香精化妆品, 2010, 2: 26-27]

9 Xiao Z, Ye L, Zhang S, Wang J, Li Y. Food Sci, 2007, 3: 155-157 (in Chinese) [肖作兵, 叶琳, 章苏宁, 王进, 李业成. 食品科学, 2007, 3: 155$157]$

10 Hu J, Zhang Y, Xiao Z, Wang X. Ind Crops Prod, 2018, 122: 85-92

11 Kuhnt T, Herrmann A, Benczédi D, Foster EJ, Weder C. Polym Chem, 2015, 6: 6553-6562

12 Treeudom T, Wanichwecharungruang SP, Seemork J, Arayachukeat S. Carbohydr Polym, 2011, 86: 1602-1609

13 Ghorani B, Tucker N. Food Hydrocolloids, 2015, 51: 227-240

14 Bollhorst T, Rezwan K, Maas M. Chem Soc Rev, 2017, 46: 2091-2126

15 Chen S, Zhao Q, Xue J, Li J, Dong J. J WuHan Univ Tech, 2006, 1: 339-342 (in Chinese) [程绍娟, 赵强, 薛建伟, 李晋平, 董晋湘. 武汉理工大 学学报, 2006, 1: 339-342]

16 Zhang X, Li H, Hou F, Dong H, Zhu Z, Cui L. J Funct Mater, 2016, 8: 8178-8181 (in Chinese) [张晓东, 李红欣, 侯扶林, 董寒, 朱正, 崔立峰. 功能材料, 2016, 8: 8178-8181]

17 Shen L, Wang G, Zheng X, Cao Y, Guo Y, Lin K, Jiang L. Chin J Catal, 2017, 38: 1373-1381 (in Chinese ) [沈丽娟, 王高杰, 郑笑笑, 曹彦宁, 郭玉峰, 林科, 江莉龙. 催化学报, 2017, 8: 1373-1381 
Wu C, Miu Y, Zhu Y, Gan N, Ou C, Cao J. Chin J Anal Chem, 2016, 12: 1820-1827 (in Chinses) [吴彩叶, 缪养宝, 朱云云, 干宁, 欧昌荣, 曹锦 轩. 分析化学, 2016, 12: 1820-1827]

19 Vaughn J, Wu H, Efremovska B, Olson DH, Mattai J, Ortiz C, Puchalski A, Li J, Pan L. Chem Commun, 2013, 49: 5724-5726

20 Hu Z, Peng Y, Kang Z, Qian Y, Zhao D. Inorg Chem, 2015, 54: 4862-4868

21 Hou L, Zhang G, Feng C, Liu X, Li D, Wang Y, Fan M. Chin J Inf Tradit Chin Med, 2016, 23: 82-86 (in Chinese) [侯莉, 张国松, 封传华, 刘晓 谦, 李东勲, 王跃生, 范玫玫. 中国中医药信息杂志, 2016, 23: 82-86]

\title{
A zirconium-based and amine-functionalized metal-organic frame- work material for adsorption and controlled release of fragrance
}

\author{
Yuhang Liu ${ }^{1,2}$, Zhuxian Zhou ${ }^{1 *}$, Liming Jiang ${ }^{2}$, Youqing Shen ${ }^{1}$ \\ ${ }^{1}$ Key Laboratory of Biomass Chemical Engineering of Ministry of Education and Center for Bionanoengineering, College of Chemical and Biological \\ Engineering, Zhejiang University, Hangzhou 310027, China \\ ${ }^{2}$ MOE Key Laboratory of Macromolecular Synthesis and Functionalization, Department of Polymer Science and Engineering, Zhejiang University, \\ Hangzhou 310027, China \\ *Corresponding author (email: zhouzx@zju.edu.cn)
}

\begin{abstract}
In this paper, we studied the adsorption and release behavior of various fragrant molecules to zirconiumbased and amine-functionalized metal-organic framework materials (MOF) UiO-66- $\mathrm{NH}_{2}$. The results showed that UiO66- $\mathrm{NH}_{2}$ has almost no difference in the adsorption of non-polar terpenoid fragrances, but there is significant difference in the adsorption of polar ester fragrance. The fragrance-adsorbed UiO-66-NH2 was characterized by SEM, PXRD and FTIR. No structural collapse and crystal form change was found after the adsorption of fragrant molecules. Amine groups in UiO-66- $\mathrm{NH}_{2}$ can form hydrogen bonds with different ester fragrance, which favor the adsorption and sustain release of fragrance. These MOF materials showed much higher adsorption capacity to fragrant molecules than the traditional adsorbent activated carbon. The release kinetics of ester fragrance was studied by headspace-gas chromatography. It was found the release kinetics was consistent with the Korsmeyer-Peppas model and the pore diffusion was the rate-limiting state of its release.
\end{abstract}

Keywords: MOF, fragrance, adsorption, control release

doi: $10.1360 / \mathrm{N} 032018-00167$ 